\title{
UNIFORM LONGITUDINAL BEAM PROFILES IN THE FERMILAB RECYCLER USING ADAPTIVE RF CORRECTION*
}

\author{
Martin $\mathrm{Hu}^{\#}$, Daniel Robert Broemmelsiek, Brian Chase, James L. Crisp, Nathan Eddy, Paul W \\ Joireman, King Yuen Ng, Fermilab, Batavia, IL, U.S.A.
}

\begin{abstract}
The Fermilab Recycler Ring is a permanent magnet based $8 \mathrm{GeV}$ anti-proton storage ring. A wideband $\mathrm{RF}$ system, driven with ARB's (ARBitrary waveform generators), allows the system to produce programmable barrier waveforms. Beam current profile distortion was observed, its origin verified both experimentally and theoretically, and an FPGA-based correction system was designed, tested and implemented to level the bunch profile.
\end{abstract}

\section{INTRODUCTION}

The Fermilab Recycler Ring is a permanent-magnet based, $8 \mathrm{GeV}$ anti-proton storage and cooling ring. Both stochastic and electron cooling have been employed to increase the phase space density to meet the collider Run$\mathrm{IIb}$ luminosity goal of $2 \mathrm{E} 32 \mathrm{~cm}^{-2} \mathrm{sec}^{-1}$. Non-uniform bunch intensities (variations up to a factor of two) cause inconsistencies in bunch-to-bunch tune shifts in the Tevatron, and made the trigger calibration challenging for the experimenters.

\section{THE ORIGIN OF BUNCH SHAPE DISTORTION}

\section{The Recycler RF system}

Details for the LLRF (Low Level RF) and HLRF (High Level RF) subsystems can be found in [2] and [3]. The low level signal from eight 8-bit DSP-controlled ARB's is sent to 4 Amplifier Research Model 3500A100 amplifiers, each capable of 3,500 watts of output power over the frequency range of $10 \mathrm{KHz}$ to $100 \mathrm{MHz}$. Each amplifier then drives a wideband RF cavity (approximately $50 \Omega$ between $100 \mathrm{KHz}$ and $20 \mathrm{MHz}$ ) in the tunnel. The voltage across the cavity gap is monitored by two 1000:1 resistive voltage dividers. It is the sum of these gap voltage monitors that provided the vital information of the actual pulse shapes the beam sees.

Apart from the RF cavities, there is a WCM (Wall Current Monitor), consisting of resistors across a ceramic gap in the beam pipe, for monitoring of the beam current profile. The WCM has a bandwidth of $3 \mathrm{KHz}$ to 100 $\mathrm{MHz}$. The low end response limit has been taken into account in our observation of the bunch shape.

\section{Recycler barrier bucket parameters}

The Recycler has three typical operational modes: Storage mode, Injection mode, and Extraction mode. In the storage mode, a pair of rectangular pulses of opposite polarity, each about $0.91 \mu$ s wide and about $2 \mathrm{kV}$ in magnitude, forms a potential well about $18.4 \mathrm{MeV}$ deep.
The bucket area is then adjusted with the separation between the two pulses. In the event of injection or extraction, beam is captured in four $2.5 \mathrm{MHz}$ bunches. In the event of an extraction for Tevatron collision, the cooled anti-proton beam, in the barrier bucket, is partitioned through a Momentum Mining process [4] for 9 transfers, with 4 bunches per transfer, sending a total of 36 bunches to the Tevatron. Any initial unevenness in the profile of the cooled anti-protons persists in the bunch shapes in the Tevatron. We will therefore focus on the storage barrier bucket distortion in the following for simplicity.

\section{Distortions in the beam current profile}

Beam in the barrier bucket were observed to display uneven profiles on the WCM. These profiles can be described as combinations of slopes and curves, which change somewhat unpredictably due to the ever changing bunch length and bunch location of the Recycler beam, environmental effects on the power amplifiers, and slow drifts in responses in time. Fig. 1 shows a typically uneven time profile of the beam, of which the frequency response of the WCM was not the main contribution to the unevenness.

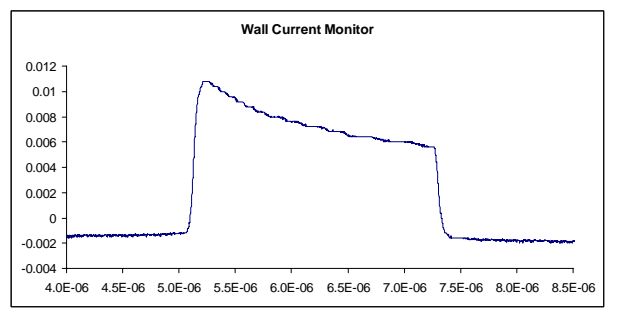

Figure 1 The Wall Current Monitor shows nonuniformity in the time profile of the beam current in the Recycler.

\section{Fidelity of the drive signal}

A pair of rectangular pulses at the output of the LLRF ARB module forming a barrier bucket was examined. The typical polar asymmetry was $0.15 \%$ of full scale, in most cases contributing insignificantly to the nonuniformity of the cold beam.

\section{Fidelity of the cavity gap resistor fanback signal: $\int V d t$}

The sum of the fanback voltages of the gap monitors was recorded with a Tektronix TDS 3054B digital oscilloscope at a time resolution of 2 ns. A total of 500 data samples were averaged to improve signal-to-noise ratio, as the distortion changed relatively slowly in 
comparison with this sampling time. A constant was added to each bin to remove any net DC component for one turn of data. The dominant feature of the processed voltage was a polar asymmetry of about $10 \%$.

The asymmetry of the positive and negative waveforms is caused by the non-symmetric saturation curves of the power amplifiers. The saturation curves are unique to each amplifier and vary with operation temperature and output power. Therefore, the static corrections that were tried first were never able to provide a workable solution for all operational conditions.

Beam loading [4] produces a linear slope on the beam profile. In this particular case it has a relatively small effect on the test beam compared to RF distortion.

Fig. 2 shows the magnified view of the bottom of the potential well derived from integrating the processed fanback voltage for one revolution period $(11.13 \mu \mathrm{s})$, super-imposed with the arbitrararily scaled WCM profile. As can be seen, the integral bears remarkable resemblence to the WCM profile.

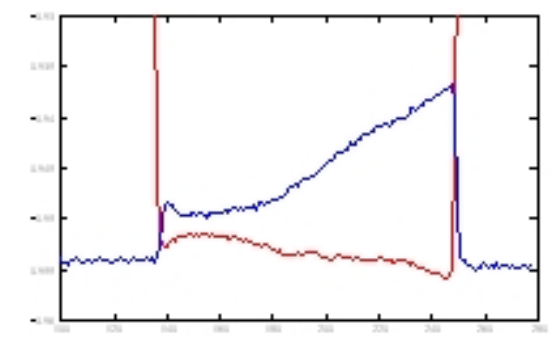

Figure 2 The magnified bottom view of the potential well (red), compared with the WCM beam profile (blue). The full scale of the potential is about $1 \mathrm{MeV}$.

\section{Theoretical verification of the observation}

A detailed account of the theoretical verification of this observation can be found in [5]. The Haissinski equation, which describes the beam current distribution in time, is

$$
\rho(\tau)=\rho(0) \exp \left[-\frac{|\mathrm{e}| \beta^{2} E_{0}}{|\eta| T_{0} \sigma_{E}^{2}} \int_{0}^{\tau} V_{\text {eff }}\left(\tau^{\prime}\right) d \tau^{\prime}\right],
$$

Here $\rho(0)$ is the ideal time profile of the beam, $\rho(\tau)$ the actual profile, $\mathrm{T}_{0}=11.13 \mu \mathrm{s}$ is the revolution period, and $\eta=-0.0085$ is the slip factor of the Recycler. The voltage $\mathrm{V}_{\text {eff }}$ was a measurement of the gap monitor voltage taken at the same time as the momentum spread, $\sigma_{E}$, was measured. A Gaussian momentum distribution was assumed. Expanding the Haissinski equation and taking the difference $\rho(\tau)-\rho(0)$, which is the net distortion on the beam current profile, we have

$$
\rho(\tau)-\rho(0)=\frac{|\mathrm{e}| \beta^{2} E_{0}}{|\eta| T_{0} \sigma_{E}^{2}} \rho(0) \int_{0}^{\tau} V_{e f f}\left(\tau^{\prime}\right) d \tau^{\prime} .
$$

It is evident from Eq. (2) that the magnitude of the distortion depends on the error voltage-time integral (the "bucket area" of the distortion), and varies inversely with the $2^{\text {nd }}$ power of the momentum spread, the effect of which has been observed in the daily operations of the
Recycler. Fig. 3 shows the super-position of the WCM profile with the computed profile, with good agreement.

\section{Experimental test of a correction}

Experiments were conducted to correct the profile distortion. The cavity gap monitor voltage was recorded

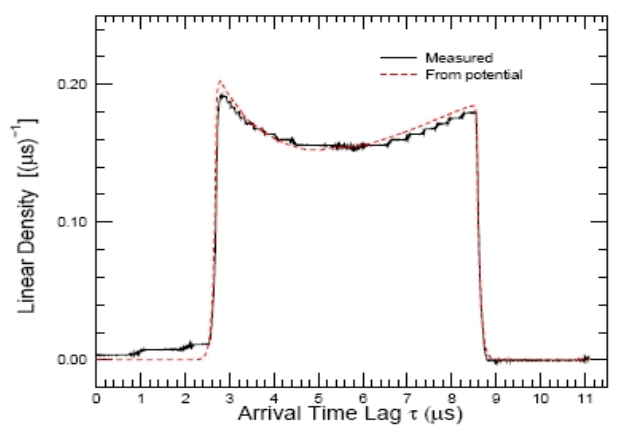

Figure 3 The super-position of the computed beam profile (dashed), based on the measured momentum spread and the Wall Current Monitor beam profile (solid).

at $2 \mathrm{~ns}$ time resolution, averaged, low-pass-filtered to remove high frequency noise, and decimated to fit the $18.936 \mathrm{~ns}$ time resolution of the ARB input tables. A difference between the measured and the ideal voltages was calculated, and an inverted version of the result was sent to the LLRF in small steps. This test was performed on a proton beam with an intensity of about $1 \times 10^{11}$, with a momentum spread of about $2 \mathrm{MeV}$, in a barrier bucket of about $2 \mu$ s long.

It can be shown that, since the RF voltage is coupled to the beam via $\mathrm{AC}$ induction, the correction waveform only contains revolution harmonics with most of the correction taking place in the first few harmonics. Fig. 4 shows the effect of one of the many successful tests of one-loop corrections. The correction was applied to flatten the beam profile, then slowly removed to recover the "curved" profile, to verify that the longitudinal emittance of the beam was not diluted to first order.

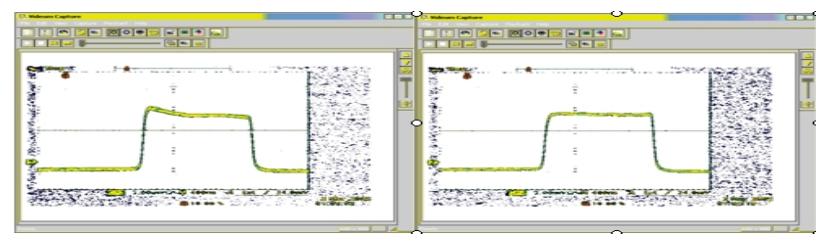

Figure 4 The WCM beam profile before a test correction (left) and after correction (right).

\section{FPGA-BASED ADAPTIVE CORRECTION}

Functionally, the correction simply generates an error signal from the difference between the reference and cavity fanback signals. This error signal is used to build a correction which is then added back to the reference signal. Any difference between the reference and the fan back signals is minimized, whether the reference signal is 
in a steady state or changing due to RF manipulations. Another remarkable feature of this algorithm is its built-in compensation of beam loading.

The adaptive correction is implemented in a custom FPGA-based Digital Damper board developed at Fermilab for digital feedback and control applications [6]. The correction system is shown in Fig. 5. The custom

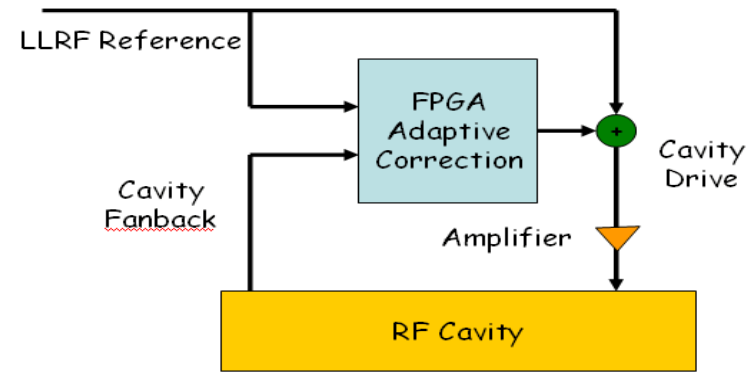

Figure 5 Block diagram of the adaptive correction system implemented into the Low Level RF.

board digitizes the Low Level RF reference and cavity fanback signals at 4 times the RF clock $(4 \times 52.811 \mathrm{MHz})$ and sends the signals to a Stratix II EP2S60 FPGA. The correction signal is then output by digital to analog converters also running at 4 times the RF clock. The correction signal is then summed back in with the LLRF reference signal by an external summing amplifier board.

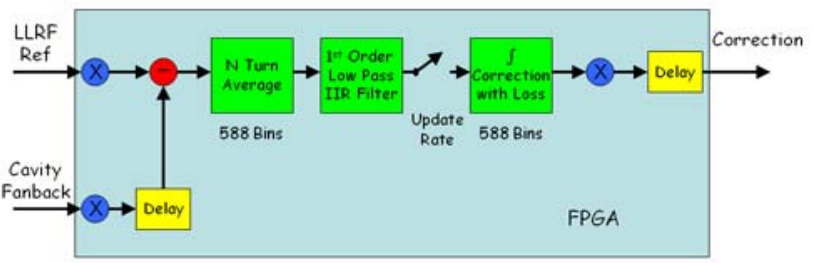

Figure 6 Block diagram of the adaptive correction algorithm implemented inside the FPGA firmware.

The algorithm to generate the correction is implemented in the custom FPGA firmware. The block diagram for the adaptive algorithm is shown in Fig. 6. The correction takes advantage of the fact that the LLRF reference signal changes very slowly, on the order of a few $\mathrm{Hz}$, and can be considered unchanging every turn. The incoming signals are summed and decimated by 4 so that the digital signal processing is done at the rate of the RF clock. Adjustable gains and a delay are used to align the fanback signal with the reference signal before taking the difference. A single turn can be divided into $588 \mathrm{RF}$ bins and the signals are treated as single turn waveforms of 588 bins sampled once per turn. This allows averaging the difference signal for up to 128 turns and integrating the difference or error in each bin to construct the correction signal. A first order IIR filter is applied to the averaged difference signal bin to bin to limit the bandwidth of the correction and prevent high frequency instabilities in the closed loop system. The IIR filter taps, the integration loss, the correction update rate, and the overall gain can be adjusted to tune the performance and stability of the system. The output delay is used to align the output correction with the reference signal into the summing amplifier.

\section{RESULTS}

The adaptive RF correction was first tested in October 2006, and has become integral with the RF system. The corrective effect on the beam profile was immediately evident. It has removed most of the profile distortion, and has reduced bunch-intensity variation from up to $200 \%$ to about $15 \%$. Studies showed that the correction produces no measurable longitudinal emittance dilution, and the system has proven to be reliable to date.

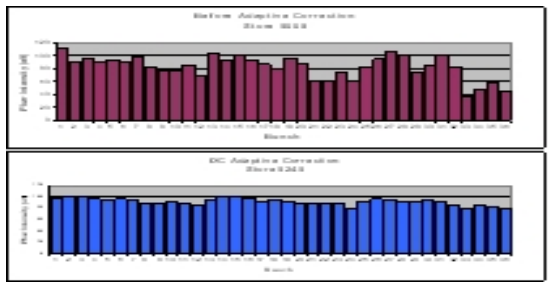

Figure 7 Bunch profiles in the Tevatron before the adaptive correction (top) and after (bottom).

\section{SUMMARY}

The non-uniform beam current profile in the Recycler has been characterized and eliminated, to a large extent, with the implementation of an FPGA-based adaptive correction. This correction has become an integral part of the Recycler Low Level RF system.

\section{REFERENCES}

[1] B. Chase, A. Mason, and K. Meisner, "Current DSP Applications in Accelerator Instrumentation and RF", Proceedings, 1997 Int. Conference on Accelerator and large Experimental Physics Control Systems, Beijing, China, 1997

[2] J.E. Dey and D.W. Wildman, "Wideband RF system for the Fermilab Recycler ring", Proceedings, 1999 Particle Accelerator Conference, pp. 869-871

[3] C.M. Bhat, "Longitudinal momentum mining of beam particles in a storage ring", Physics Letters A, Volume 330, Issue 6, 4 October 2004, pp. 481-486

[4] C.M. Bhat and K.Y. Ng, Potential-Well Distortion in Barrier RF, Proc. 30th Advanced ICFA Beam Dynamics Workshop on High Luminosity $\mathrm{e}+\mathrm{e}-$ Collisions (e+e- Factories 2003), Stanford, CA Oct. 13-16, 2003

[5] J. Crisp, M. Hu and K.Y. Ng, "Correction of Unevenness in Recycler Beam Profile", Proceedings, HB2006, Tsukuba, Japan, pp. 244-246

[6] N. Eddy and J. Crisp, "Transverse Digital Damper System for the Fermilab Anti-proton Recycler", Proceedings, 12th Beam Instrumentation Workshop (BIW06), Fermilab, Batavia, Illinois, May 2006, pp. 293-297 